\title{
SUBTROCHANTERIC FRACTURES IN OSTEOMALACIA
}

\author{
J. Chalmers, Edinburgir, Scotland
}

The eccentric load transmitted by the femoral shaft during weight-bearing concentrates very high stresses in the upper third of the femoral shaft, particularly on its medial part. According to Koch (1917) a vertical load of 100 pounds (45.36 kilograms) causes compression stresses in this area exceeding 1,000 pounds per square inch $(70.307 \mathrm{~kg} /$ square centimetre) (Fig. 1).

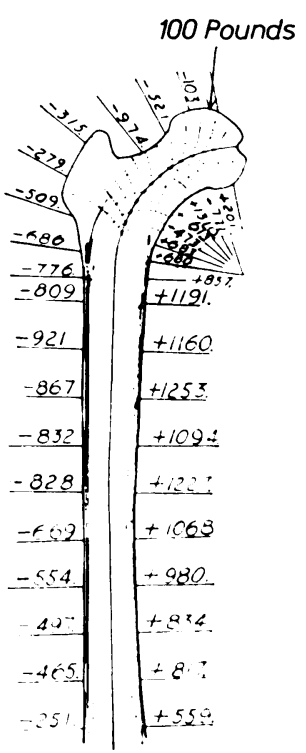

FIG. 1

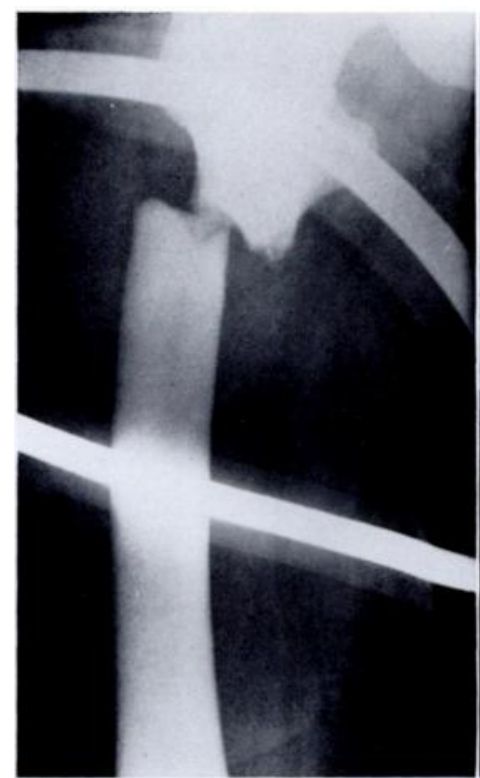

FIG. 2

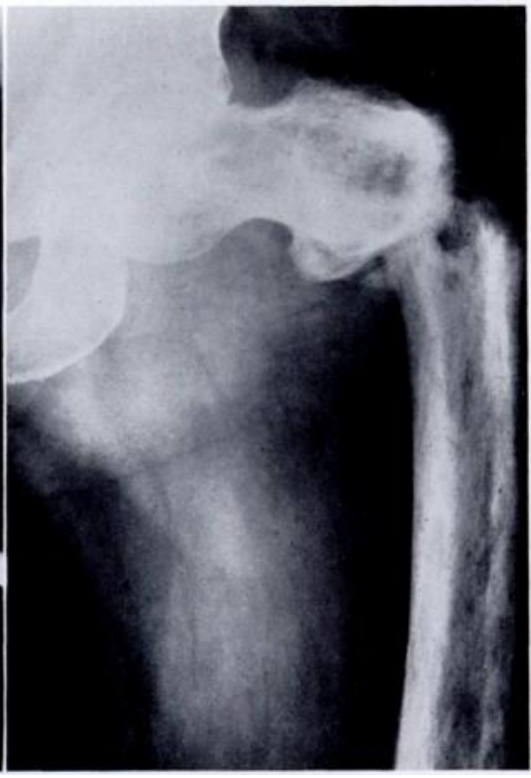

FIG. 3

Figure 1-Calculation of stresses developing in a femur when subjected to a vertical load of 100 pounds $(45 \cdot 36$ kilograms). The highest compression stresses develop in the medial cortex in the upper third of the shaft. (After Koch, J. C., 1917.) - sign indicates compressive stress: - sign indicates tensile stress. Only maximum stresses are shown (in pounds per square inch). Figure 2-Radiograph of a pathological subtrochanteric fracture in osteoporosis. Figure 3-A similar fracture in Paget's disease.

In normal circumstances the bone in this area is, of course, adapted to withstand such loads, and subtrochanteric fractures are usually the result of considerable violence. However, any disorder affecting bone strength reveals the vulnerability of this site, and pathological subtrochanteric fractures are well recognised in such disorders as Paget's disease, renal osteodystrophy, osteogenesis imperfecta and osteopetrosis (Figs. 2 and 3).

The purpose of this paper is to draw attention to the frequency with which subtrochanteric fractures occur in patients suffering from osteomalacia and to point out the difficulties of internal fixation of these fractures because of the softness of the bone.

\section{CASE REPORTS}

Case 1-A woman aged eighty-two was admitted to hospital for investigation of skeletal pain and muscular weakness of seven years' duration. One week before admission she sustained fractures of both bones of the left forearm. The diagnosis of osteomalacia was made on the basis of skeletal tenderness, widespread Looser`s zones throughout the skeleton, serum calcium 


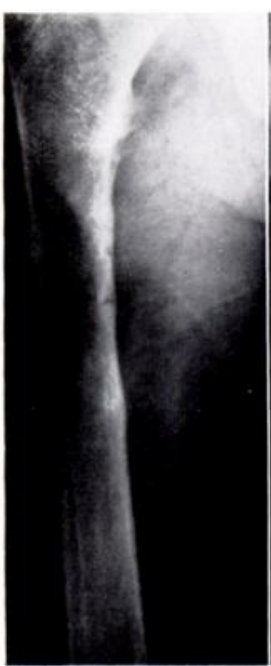

FIG. 4

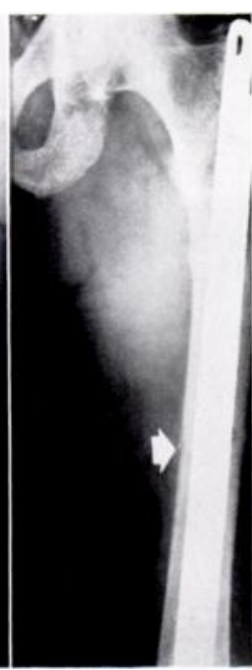

FIG. 5
Case 1. Figure 4-Radiograph of the right femur showing several Looser's zones in the medial cortex. Figure 5The left femur sustained a complete fracture through a similar Looser's zone (indicated by the arrow). Internal fixation with two intramedullary nails achieved satisfactory stabilisation.

of 6.2 milligrams per cent, serum phosphate of 2.4 milligrams per cent and alkaline phosphatase of $36 \mathrm{KA}$ units. The patient's diet had been deficient in vitamin $D$, and pancreatic function tests indicated pancreatic insufficiency. While being lifted from a trolley to her bed she sustained a fracture through the upper third of the left femoral shaft at the site of a pre-existing Looser's zone (Figs. 4 and 5). The fracture was stabilised by intramedullary nailing. two nails being required because of the wide medullary canal. She was given supplements of vitamin D. initially 100,000 IU per day, later reduced to $50,000 \mathrm{IU}$ weekly. The fracture healed and the osteomalacia was cured according to clinical, biochemical and radiological evidence. Two years later she was well and active and had lost all skeletal pain and weakness.

Case 2-A woman aged sixty-nine, with a history of partial gastrectomy twelve years before and right hemiplegia six years before, sustained a subtrochanteric fracture of the right femur in a fall. The diagnosis of osteomalacia was indicated by a serum calcium level of 8 milligrams per cent, serum phosphate of 2.8 milligrams per cent and alkaline phosphatase of $29 \mathrm{KA}$ units.

The fragments were fixed with a nail and five-hole plate but the nail and plate cut out two weeks later (Figs. 6 and 7). A seven-hole plate was applied and the limb supported with

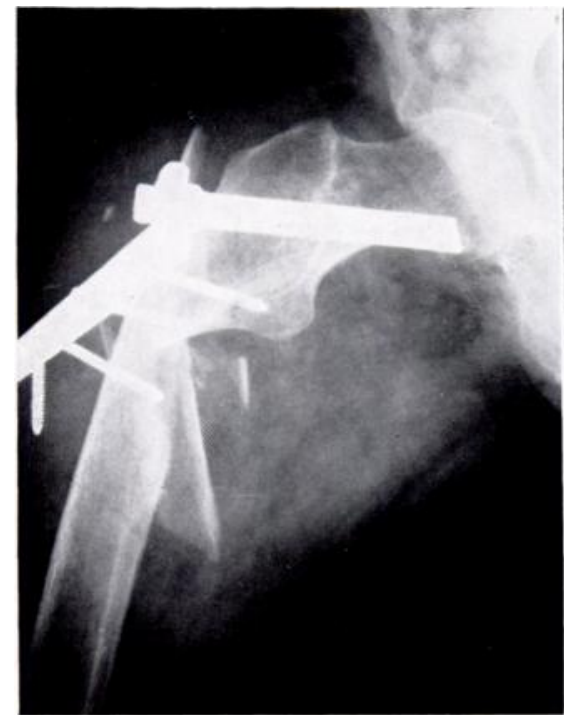

FiG. 6

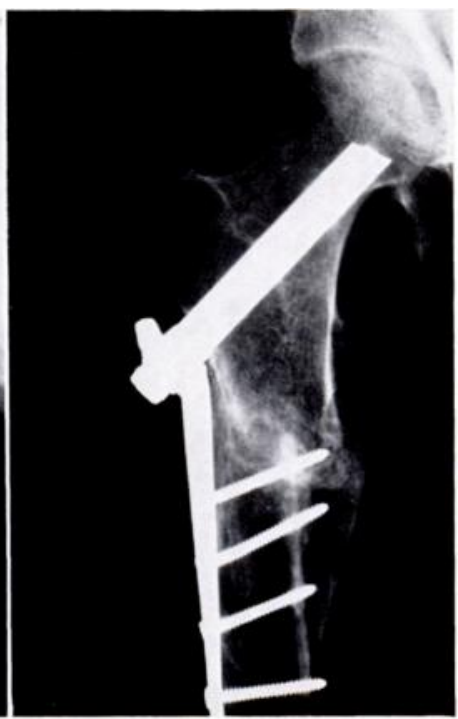

FIG. 7

Case 2. Figure 6-This subtrochanteric fracture was initially fixed with a pin and five-hole plate which cut out two weeks later. Figure 7-A seven-hole plate supplemented with Hamilton Russell traction allowed healing to take place.

Hamilton Russell traction. Vitamin D was given, initially in doses of 50,000 IU per day, later reduced to 50,000 IU per week. The fracture healed rapidly and the serum values of calcium and phosphate returned to normal. 
Case 3-A woman aged eighty-five who gave a history of pain in the legs and difficulty with walking for several years developed a spontaneous subtrochanteric fracture of the left femur. The diagnosis of osteomalacia was based on the presence of numerous Looser's zones on

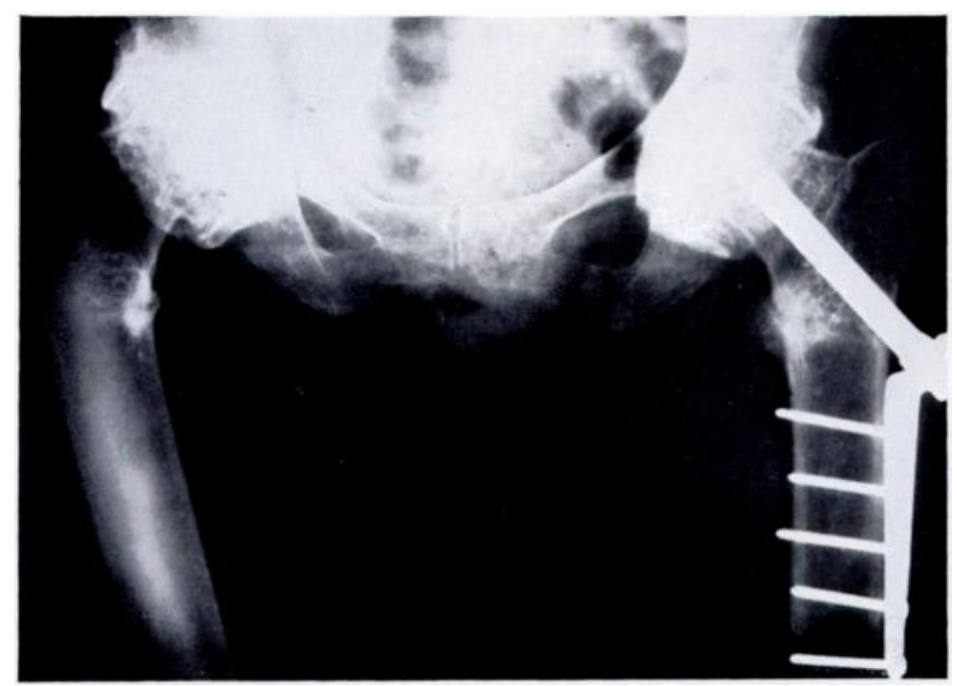

FIG. 8

Case 3-Note the symmetrical Looser's zones at the level of the lesser trochanters. A complete fracture developed at this site on the left.

skeletal radiography, one being present in the opposite femur at the level of the fracture (Fig. 8). The diagnosis was further supported by biochemical abnormalities, notably a serum calcium level of 8.7 milligrams per cent, phosphate of 1.9 milligrams per cent, and alkaline phosphatase of $33 \mathrm{KA}$ units. Bone biopsy showed a marked excess of osteoid (22 per cent of the total bone area). The etiology of the osteomalacia was not established, but previous cholecystectomy and long-standing peptic ulceration may have been factors. Internal fixation with a nail and five-hole plate was carried out and vitamin D therapy, 50,000 IU per day, instituted. The fracture healed rapidly and the osteomalacia was cured as shown by clinical and biochemical evidence.

Case 4-A woman aged sixty-three had been housebound for years because of skeletal pain and muscular weakness. A spontaneous subtrochanteric fracture of the right femur occurred while she was attempting to walk. The diagnosis of osteomalacia was based on the presence of numerous Looser's zones in radiographs including one in the opposite femur at the same site as the fracture. Serum calcium was 8.1 milligrams per cent, phosphate 1.1 milligrams per cent and alkaline phosphatase $25 \mathrm{KA}$ units. Enquiry into her diet revealed a gross deficiency of vitamin $D$. A nail used with a seven-hole plate for fixation cut out one week later (Fig. 9) and was replaced by an intramedullary nail. Vitamin D, 50,000 IU per day, was given and the fracture healed readily. Her general health improved

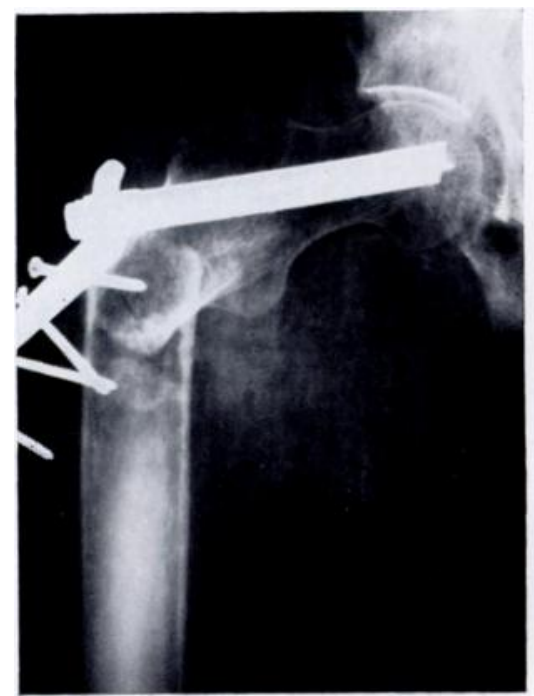

Fig. 9

Case 4-A pin and seven-hole plate proved inadequate to stabilise this fracture. Subsequent fixation with an intramedullary nail was successful. 
dramatically as her osteomalacia disappeared, and from being a housebound invalid she became fully active once more.

Case 5-A woman aged eighty-five who suffered from senility was referred from her geriatric home because of inability to walk for two months. Radiographs showed a subtrochanteric fracture of the right femur and multiple Loosers zones elsewhere. The diagnosis of osteomalacia was confirmed by a serum calcium level of 7.9 milligrams per cent, phosphate of 1.9 milligrams per cent and alkaline phosphatase of $52 \mathrm{KA}$ units. Internal fixation with a pin and seven-hole plate was carried out and vitamin $D$ in doses of $50,000 \mathrm{IU}$ per day was begun. The patient died three weeks later of respiratory infection. Permission for necropsy was not granted.

Case 6-A woman aged eighty-nine had suffered from leg pain and weakness for years. She sustained a subtrochanteric fracture of the left femur in a fall (Fig. 10). Radiographs showed multiple Looser's zones throughout the skeleton. The diagnosis of osteomalacia was

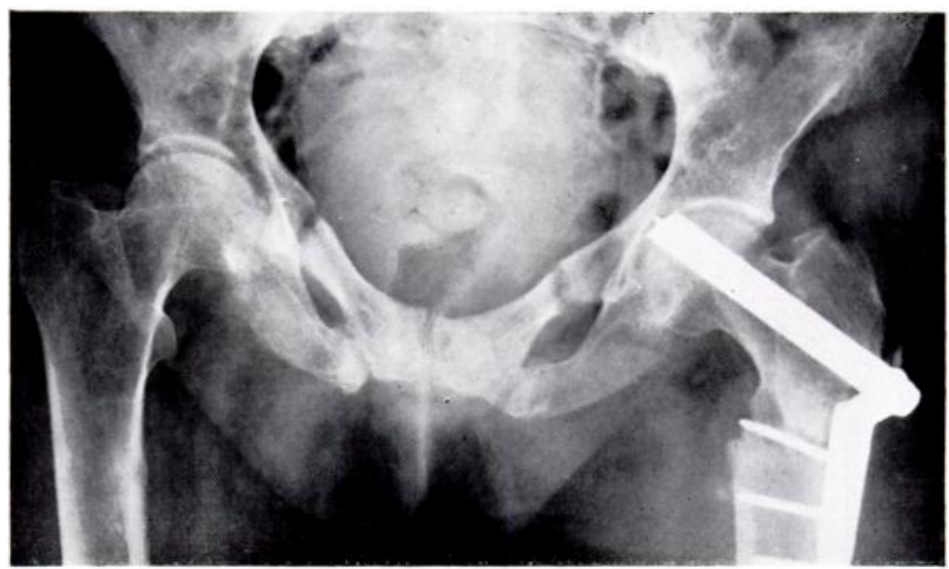

Fig. 10

Case 6-A pin and seven-hole plate gave adequate stabilisation in this high subtrochanteric fracture. Note the symmetrical Looser's zones in the superior pubic rami.

confirmed by a serum calcium level of 8 milligrams per cent, phosphate of 2.5 milligrams per cent, alkaline phosphatase $23 \mathrm{KA}$ units and excess of osteoid on bone biopsy. Evidence of intestinal malabsorption was found. The fracture was internally fixed with a nail and seven-hole plate, and vitamin D supplements of $50,000 \mathrm{IU}$ per day were given. The fracture healed readily and her activity increased greatly as her osteomalacia subsided.

\section{DISCUSSION}

The high stress to which the upper third of the femoral shaft is subjected predisposes to pathological fracture at this site from any of the wide range of local or systemic disorders that weaken bone structure.

The deficiency of mineralisation that characterises osteomalacia results in a reduction in the compression strength of bone. Looser's zones are most often seen in areas of cortical bone subjected to compression stress, the upper medial femoral cortex being a particularly common site (Chalmers, Conacher, Gardner and Scott 1967). In four of the six cases of subtrochanteric fracture reported here there was evidence that such a lesion preceded the development of the complete fracture.

It is important that all patients presenting with pathological subtrochanteric fracture should be examined for osteomalacia unless some other obvious pathology is recognised. 
Since osteomalacia in this country most commonly affects elderly women (Chalmers 1969), the most important disease from which it must be distinguished is senile osteoporosis, which occurs predominantly in the same age group. The distinction is not merely of academic interest, because osteomalacia is readily cured by supplements of vitamin $\mathrm{D}$, whereas no treatment is known which markedly influences the course of senile osteoporosis. The diagnosis of osteomalacia is not difficult provided that one remains constantly alert to the possibility of its presence. This awareness should be aroused by any pathological fracture occurring in the elderly patient. In a survey of 130 patients with fractures of the neck and trochanteric region of the femur 12 per cent were found to have significant osteomalacia (Chalmers, Barclay, Davison, MacLeod and Williams 1969). Other features such as bone pain and tenderness and muscular weakness should also suggest the diagnosis. The diagnosis may be established by the presence of Looser's zones elsewhere in the skeleton, by low levels of serum calcium and phosphate, and by elevation of the serum alkaline phosphatase. Bone biopsy will confirm the diagnosis when there is doubt. Looser's zones were present in five of the six patients reported here and all showed gross abnormalities in the biochemical values.

There is general agreement that subtrochanteric fractures are best treated by internal fixation but less agreement about which technique should be used. The softness of the bone in osteomalacia affords a poor purchase for screws and in two cases failure of fixation occurred with the use of conventional nail and plates. Intramedullary nails provided adequate fixation in the two cases in which they were used. This experience suggests that intramedullary fixation gives more reliable stabilisation when dealing with soft bone. It is of course necessary to treat the underlying osteomalacia with supplements of vitamin D appropriate to the etiology of the disease, because otherwise fractures in osteomalacia may not heal (Chalmers et al. 1967). One of the most rewarding aspects of these cases has been the marked improvement in general health and increased function which the patients gained from cure of their osteomalacia. In so far as a fracture leads to the diagnosis of the underlying condition, it may be regarded by the patient as a lucky event.

\section{REFERENCES}

Chalmers, J. (1968): Osteomalacia: A Review of 93 Cases. Jourrial of the Royal College of Surgeons of Edinburgh, $13,255$.

Chalmers, J., Barclay, A., Davison, A. M., Macleod, D. A. D., and Williams, D. A. (1969): Quantitative Measurements of Osteoid in Health and Disease. Clinical Orthopaedics, 63, 196.

Chalmers, J., Conacher, W. D. H., Gardner, D. L., and Scotr, P. J. (1967): Osteomalacia-A Common Disease in Elderly Women. Journal of Bone and Joint Surgery, 49-B, 403.

Косн, J. C. (1917): The Laws of Bone Architecture. American Journal of Anatomy, 21, 177.

VOL. 52 B, No. 3, AUGUSt 1970 